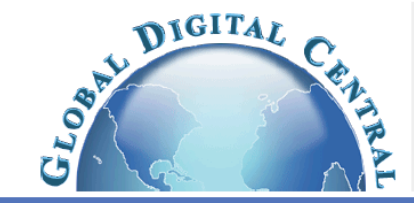

Frontiers in Heat and Mass Transfer

Available at www.ThermalFluidsCentral.org

\title{
CHEMICAL REACTION AND RADIATION EFFECTS ON NATURAL CONVECTION IN POROUS MEDIUM SATURATED WITH POWER-LAW FLUID
}

\author{
D. Srinivasacharya* and G. Swamy Reddy \\ Department of Mathematics, National Institute of Technology, Warangal, Andhra Pradesh, 506004, India
}

\begin{abstract}
The natural convection heat and mass transfer along a vertical plate embedded in non-Newtonian Power-law fluid saturated porous medium in the presence of first order chemical reaction and radiation is studied. The governing partial differential equations are transformed into ordinary differential equations using similarity transformations. The resulting equations are solved numerically using Shooting method. The effect of radiation parameters and chemical reaction parameter and power law index on non-dimensional velocity, temperature and concentration fields are discussed. The variation of different parameters on heat and mass transfer rates is presented in tabular form.
\end{abstract}

Keywords: Natural convection; Porous media; Chemical reaction; Radiation; Power-law fluid; Heat and Mass transfer.

\section{INTRODUCTION}

Coupled heat and mass transfer by natural convection in a fluid-saturated porous medium has gained much attention from the researchers because of its engineering and industrial applications. These applications include design of chemical processing equipment, formation and dispersion of fog, distributions of temperature and moisture over agricultural fields and groves of fruit trees and damage of crops due to freezing and pollution of the environment etc. A number of studies have been reported in the literature focusing on the problem of combined heat and mass transfer in porous media. A review of convective heat transfer in porous medium is presented in the book by Nield and Bejan (2006). The majority of these studies dealt with the traditional Newtonian fluids. It is well known that most fluids which are encountered in chemical and allied processing applications do not satisfy the classical Newton's law and are accordingly known as non-Newtonian fluids. Due to the importance of the applications of non-Newtonian fluids in biology, physiology, technology, and industry, considerable efforts have been directed towards the analysis and understanding of such fluids. A number of mathematical models have been proposed to explain the rheological behavior of non-Newtonian fluids. Among these, a model which has been most widely used for nonNewtonian fluids, and is frequently encountered in chemical engineering processes, is the power-law model. Although this model is merely an empirical relationship between the stress and velocity gradients, it has been successfully applied to non-Newtonian fluids experimentally.

Radiation effects on convective heat transfer problems have assumed an increasing importance in electrical power generation, astrophysical flows, solar power technology, space vehicle re-entry and other industrial areas. Since the solution for convection and radiation equation is quite compli- cated, there are few studies about simultaneous effect of convection and radiation for internal flows. On the other hand, chemical reaction effects on heat and mass transfer are of considerable importance in hydrometallurgical industries and chemical technology. Research on combined heat and mass transfer with chemical reaction and thermophoresis effect can help to design for chemical processing equipment, formation and dispersion of fog, distribution of temperature and moisture over agricultural fields as well as groves of fruit trees, damage of crops due to freezing, food processing, cooling towers, chemically-reactive vapour deposition boundary layers in optical materials processing etc. Several investigators have examined the effect of chemical reaction on the flow, heat and mass transfer past a vertical plate.

Free convection from a horizontal line heat source in a power-law fluid-saturated porous medium was studied by Nakayama (1993). The study of free convection in boundary layer flows of power law fluids past a vertical flat plate with suction/injection was done by Sahu and Mathur (1996). Free convection heat and mass transfer of non-Newtonian power law fluids with yield stress from a vertical flat plate in a saturated porous media was studied by Rami and Arun (2000). Buoyant convection of power-law fluid in an enclosure filled with heat-generating porous media was considered by Hyun and Kim (2004). Prabhu et al. (2005) have considered the effects of chemical reaction, heat and mass transfer on MHD flow over a vertical stretching surface with heat source and thermal stratification effects. Salem (2006) has considered the coupled heat and mass transfer in Darcy-Forchheimer mixed convection from a vertical flat plate embedded in a fluid saturated porous medium under the effects of radiation and viscous dissipation. Ghosh and Pop (2007) have consid-

*Corresponding author. Email: dsc@nitw.ac.in, dsrinivasacharya@yahoo.com 
ered thermal radiation of an optically thick gray gas in the presence of indirect natural convection by employing Rosseland approximation for diffusion concept of radiation heat transfer. Postelnicu (2007) studied the influence of chemical reaction on heat and mass transfer by natural convection from vertical surfaces in porous media by considering Soret and Dufour effects. Orhan Aydn (2008) have considered Chemical reaction effects on heat and mass transfer with radiation are of considerable importance in hydrometallurgical industries and chemical technology such as polymer production and food processing. Transient analysis of heat and mass transfer by natural convection in power law fluid past a vertical plate immersed in a porous medium is studied by Elgazery (2008). Free convection heat transfer from a vertical flat plate embedded in a thermally stratified non-Newtonian fluid saturated non-Darcy porous medium is analyzed by Kairi and Murthy (2009). Hayat et al. (2010) have considered the Effects of radiation and magnetic field on the mixed convection stagnation-point flow over a vertical stretching sheet in a porous medium. Unsteady natural convective power-law fluid flow past a vertical plate embedded in a non-Darcian porous medium in the presence of a homogeneous chemical reaction was studied recently by Chamkha et al. (2010). Gaied and Eid (2011) presented a numerical analysis of the free convection coupled heat and mass transfer for non-Newtonian power-law fluids with the yield stress flowing over a two-dimensional or axisymmetric body of an arbitrary shape in a fluid-saturated porous medium. Recently, Aouachria et al. (2012) studied the double diffusion effects on convection in flow on vertical plate imbedded in porous media.

From the literature survey, it seems that the problem of natural convection heat and mass transfer from vertical plate in Darcy porous media saturated in power-law fluid in presence of radiation and chemical reaction effects have not been investigated so far. Thus this work aims to study the effects of Radiation and Chemical reaction on natural convection in a power-law fluid embedded in a Darcy porous medium with variable surface temperature and concentration.It is established that similarity solutions are possible only when the variation in the temperature and concentration are linear functions of the distance from the leading edge measured along the plate. Using these similarity transformations, the governing system of partial differential equations is transformed into a system of non-linear ordinary differential equations and then solved numerically using shooting method along with fourth order Runge-Kutta integration.

\section{MATHEMATICAL FORMULATION}

Consider the natural convection heat and mass transfer along a vertical plate in a non-Newtonian power-law fluid saturated Darcy porous medium. Choose the coordinate system such that $\mathrm{x}$-axis is along the vertical plate and y-axis normal to the plate. The plate is maintained at variable temperature and concentration, $T_{w}(x)$ and $C_{w}(x)$, respectively. The temperature and concentration of the ambient medium are $T_{\infty}$ and $C_{\infty}$ respectively is shown in Fig.1. Assume that the fluid and the porous medium have constant physical properties except for the density variation required by the Boussinesq approximation. The flow is steady, laminar, two dimensional. The porous medium is isotropic and homogeneous. The fluid and the porous medium are in local thermodynamical equilibrium. The fluid is considered to be a gray, absorbing emitting radiation but nonscattering medium. The optically thick radiation limit is considered in the present study where the radiative heat flux term can be simplified by using the Rosseland approximationand (Sparrow and Cess, 1978). The radiative heat flux in the $x$-direction is considered negligible in comparison to the $y$-direction. This approximation is valid at points far from the boundary surface, and is good for intensive absorption (Hossain et al., 2001, Kuznetsov and Nield, 2010). Also, it is assumed that there exists a homogenous chemical reaction of first-order with rate constant $k_{1}$ between the diffusing species and the fluid.

Using the Boussinesq and boundary layer approximations, the gov-

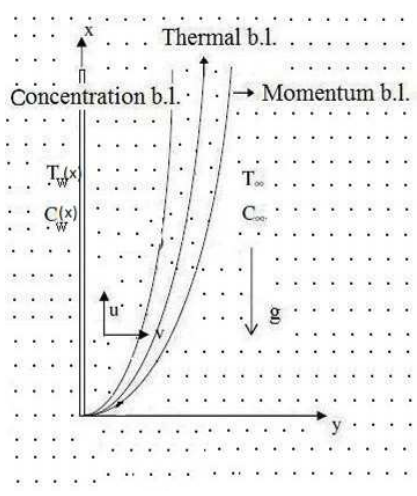

Fig. 1 Flow model and physical coordinate system

erning equations for the power law fluid are given by

$$
\begin{gathered}
\frac{\partial u}{\partial x}+\frac{\partial v}{\partial y}=0 \\
u^{n}=\frac{g K}{\nu}\left\{\beta_{T}\left(T-T_{\infty}\right)+\beta_{C}\left(C-C_{\infty}\right)\right\} \\
u \frac{\partial T}{\partial x}+v \frac{\partial T}{\partial y}=\frac{\partial}{\partial y}\left\{\alpha_{m} \frac{\partial T}{\partial y}-\frac{1}{\rho C_{p}} q_{y}^{r}\right\} \\
u \frac{\partial C}{\partial x}+v \frac{\partial C}{\partial y}=D_{m} \frac{\partial^{2} C}{\partial y^{2}}-k_{1}\left(C-C_{\infty}\right)
\end{gathered}
$$

where $u$ and $v$ are the Darcian velocity components along $x$ and $y$ directions, $T$ is the temperature, $C$ is the concentration, $\nu$ is the kinematic viscosity, $K$ is the Darcy permeability, $g$ is the acceleration due to gravity, $\beta_{T}$ is the coefficient of thermal expansion and $\beta_{C}$ is the coefficient of concentration expansion, $\alpha_{m}$ is the thermal diffusivity, $D_{m}$ is the mass diffusivity of the medium, $C_{p}$ is the specific heat capacity, $k_{1}$ is the rate of chemical reaction, $q_{y}^{r}$ is the radiative heat flux term and $n$ is the power-law index.When $n=1$, the Eq. (2) represents a Newtonian fluid. Therefore, deviation of $\mathrm{n}$ from a unity indicates the degree of deviation from Newtonian behavior. For $n<1$, the fluid is pseudo plastic and for $n>1$, the fluid is dilatant.

The boundary conditions are given by

$$
\begin{aligned}
& v=0, T=T_{w}(x)=T_{\infty}+E x^{l}, C=C_{w}(x)=C_{\infty}+F x^{m} \text { at } y=0 \\
& u \rightarrow 0, \quad T \rightarrow T_{\infty}, \quad C \rightarrow C_{\infty} \quad \text { as } \quad y \rightarrow \infty
\end{aligned}
$$

where $E, F, l$, and $m$ are constants.

The quantity $q_{r}$ on the right-hand side of equation (3) represents the radiative heat flux in the $y$ direction. In order to reduce the complexity of the problem, a more detailed representation for the radiative heat flux, for optically thick radiation limit, is considered in the present analysis. Thus, the radiative heat flux term in the energy equation is simplified by utilizing the Rosseland diffusion approximation (Sparrow and Cess 1978) for an optically thick boundary layer as follows:

$$
q_{r}=-\frac{4 \sigma^{*}}{3 k^{*}} \frac{\partial T^{4}}{\partial y}
$$

where $\sigma^{*}$ and $k^{*}$ are the Stefan-Boltzmann constant and the mean absorption coefficient respectively.

We assume that the differences of the temperature within the flow are sufficiently small such that $T^{4}$ may be expressed as a linear function 
of the temperature. This is accomplished by expanding in a Taylor series about $T_{\infty}$ and neglecting higher-order terms, thus

$$
T^{4} \cong 4 T_{\infty}^{3} T-3 T_{\infty}^{4}
$$

Using Eqs.(6) and (7) in the last term of Eq.(3), we obtain

$$
u \frac{\partial T}{\partial x}+v \frac{\partial T}{\partial y}=\alpha_{m} \frac{\partial^{2} T}{\partial y^{2}}+\frac{16 \sigma^{*} T_{\infty}^{3}}{3 \rho C_{p} k^{*}} \frac{\partial^{2} T}{\partial y^{2}}
$$

\section{SOLUTION OF THE PROBLEM}

The continuity equation (1) is satisfied by introducing the stream function $\psi$ such that

$$
u=\frac{\partial \psi}{\partial y}, \quad v=-\frac{\partial \psi}{\partial x}
$$

In order to explore the possibility for the existence of similarity, we assume

$$
\left.\begin{array}{c}
\psi=A x^{a} f(\eta), \quad \eta=B y x^{b}, \\
\frac{T-T_{\infty}}{T_{w}(x)-T_{\infty}}=\theta(\eta), \quad T_{w}(x)-T_{\infty}=E x^{l} \\
\frac{C-C_{\infty}}{C_{w}(x)-C_{\infty}}=\phi(\eta), \quad C_{w}(x)-C_{\infty}=F x^{m}
\end{array}\right\}
$$

where $A, B, a$, and $b$ are constants. Substituting (9) and (10) in (2), (4) and (8), it is found that similarity exists only if $a=1, b=0, l=m=n$. Hence, appropriate similarity transformations are

$$
\left.\begin{array}{c}
\eta=B y, \quad \psi=A x f(\eta), \\
\frac{T-T_{\infty}}{T_{w}(x)-T_{\infty}}=\theta(\eta), \quad T_{w}(x)-T_{\infty}=E x^{n} \\
\frac{C-C_{\infty}}{C_{w}(x)-C_{\infty}}=\phi(\eta), \quad C_{w}(x)-C_{\infty}=F x^{n}
\end{array}\right\}
$$

Making use of the similarity transformations (11) in (2), (4) and (8) we get the following nonlinear system of differential equations. .

$$
\begin{gathered}
\left(f^{\prime}\right)^{n}=[\theta+N \phi] \\
\theta^{\prime \prime}=\frac{1}{\left[1+\frac{4}{3} R\right]}\left[n f^{\prime} \theta-f \theta^{\prime}\right] \\
\phi^{\prime \prime}=\operatorname{Le}\left[n f^{\prime} \phi-f \phi^{\prime}+\gamma \phi\right]
\end{gathered}
$$

where the prime denotes differentiation with respect to $\eta$ alone.

$N=\frac{\beta_{C} F}{\beta_{T} E}$ is the buoyancy parameter, $\gamma=\frac{k_{1}}{B^{2} \alpha_{m}}$ is the chemical reaction parameter, $R=\frac{4 \sigma T_{\infty}^{3}}{k^{*} k}$ is the conduction radiation parameter, $L e=\frac{\alpha_{m}}{D_{m}}$
is the Lewis number.

Making the use of dimensional analysis, we get

$$
A=\left[\frac{g K E \beta_{T} \alpha_{m}^{n}}{\nu}\right]^{\frac{1}{2 n}}, \quad B=\left[\frac{g K E \beta_{T}}{\nu \alpha_{m}^{n}}\right]^{\frac{1}{2 n}}
$$

Boundary conditions (5) in terms of $f, \theta$ and $\phi$ become

$$
\begin{aligned}
& f=0, \quad \theta=1, \quad \phi=1, \quad \text { at } \quad \eta=0 \\
& f^{\prime}=0, \quad \theta=0, \quad \phi=0 \quad \text { as } \quad \eta \rightarrow \infty
\end{aligned}
$$

The parameters of engineering interest for the present problem are the Nusselt and Sherwood numbers, which are given by

$$
\frac{N u_{x}}{B x}=-\theta^{\prime}(0), \quad \frac{S h_{x}}{B x}=-\phi^{\prime}(0)
$$

\section{RESULTS AND DISCUSSION}

The flow equation (12) coupled with the energy and concentration equations (13) and (14) constitute a set of nonlinear non-homogeneous differential equation for which closed-form solution cannot be obtained and hence the problem has to be solved numerically. The boundary value problem given by equations (12)-(14) along with the boundary conditions (15) are solved using the Shooting method by giving appropriate initial guess values for $f^{\prime}(0), \theta^{\prime}(0)$ and $\phi^{\prime}(0)$ to match the values with the corresponding boundary conditions at $f^{\prime}(\infty), \theta(\infty)$ and $\phi(\infty)$ respectively. In the present study, the boundary conditions for $\eta$ at $\infty$ varies with parameter values and it has been suitably chosen at each time such that the velocity, temperature and concentration profiles approach zero at the outer edge of the boundary layer. Extensive calculations have been performed to obtain the wall velocity, temperature and concentration fields for a wide range of parameters. The effects of radiation, chemical reaction and power-law index parameter are studied.

The non-dimensional velocity $f^{\prime}(\eta)$, temperature $\theta(\eta)$ and concentration $\phi(\eta)$ are plotted for $N=1, L e=1, \gamma=1$ in Figs. 2(a)2 (c) with varying radiation parameter by considering pseudo-plastic fluids with $n=0.5$. It is observed from Fig.2(a) that the velocity of the fluid increased with increasing the value of radiation parameter. It is noted from Fig.2(b) that the temperature of the fluid is increased with the increase in the value of the radiation parameter. It is found from Fig.2(c) that the concentration of the fluid is decreased with increase in the value of the radiation parameter. These results can be explained by the fact that an increase in the radiation parameter $R=\frac{4 \sigma^{*} T_{\infty}^{3}}{k^{*} k}$ for given $k$ and $T_{\infty}$ means decrease in the Rosseland radiation absorptivity $k^{*}$. Hence, the divergence of radiative heat flux $q_{r}$ increases as $k^{*}$ decreases. Therefore, the rate of radiative heat transferred to the fluid increases and consequently the fluid temperature and along with the velocity of its particle also increases.

The variation of the non-dimensional velocity $f^{\prime}(\eta)$, temperature $\theta(\eta)$ and concentration $\phi(\eta)$ are plotted for $N=1, L e=1, \gamma=1$ with varying radiation parameter by considering Newtonian fluids with $n=1.0$ is shown in Figs.3(a)-3(c). It is observed from Fig.3(a) that the velocity of the fluid increased with increasing the value of the radiation parameter. It is found from Fig.3(b) that the increase in the value of the radiation parameter increases the temperature of the fluid in the medium. It is seen from Fig.3(c) that the concentration of the fluid is decreased by increasing the value of the radiation parameter. Increasing the thermal radiation parameter produces an increase in the thermal condition of the fluid and its thermal boundary layer. More flow is induced in the boundary layer by the increase in the fluid temperature thereby causing the velocity of the fluid to increase as well.

The effect radiation parameter on the non-dimensional velocity $f^{\prime}(\eta)$, temperature $\theta(\eta)$ and concentration $\phi(\eta)$ for $N=1, L e=1, \gamma=1$ is depicted in Fig.4(a)-4(c) in the presence of dilatant fluids with $n=1.5$. It is observed from Fig.4(a) that the fluid velocity is increased with increase in the value of radiation parameter. It is seen from Fig.4(b) that the temperature of the fluid in the medium is increased with increase in the value of the radiation parameter. This result qualitatively agrees with expectations since the effect of radiation and surface temperature are to increase the rates of energy transport to the fluid, thus increasing the temperature of the fluid. It is found from Fig.4(c) the concentration of the fluid is decreased by increasing the value of the Radiation parameter. The presence of thermal radiation enhances the thermal state of the fluid causing its temperature to increase and the solute concentration to decrease.

The non-dimensional velocity $f^{\prime}(\eta)$, temperature $\theta(\eta)$ and concentration $\phi(\eta)$ are plotted for $N=1, L e=0.5, R=0.5$ in Figs.5(a)-5(c) with varying chemical reaction parameter by considering pseudo-plastic fluids with $n=0.5$. It is observed from Fig.5(a) that the velocity of the fluid decreased with increasing the value of chemical reaction parame- 


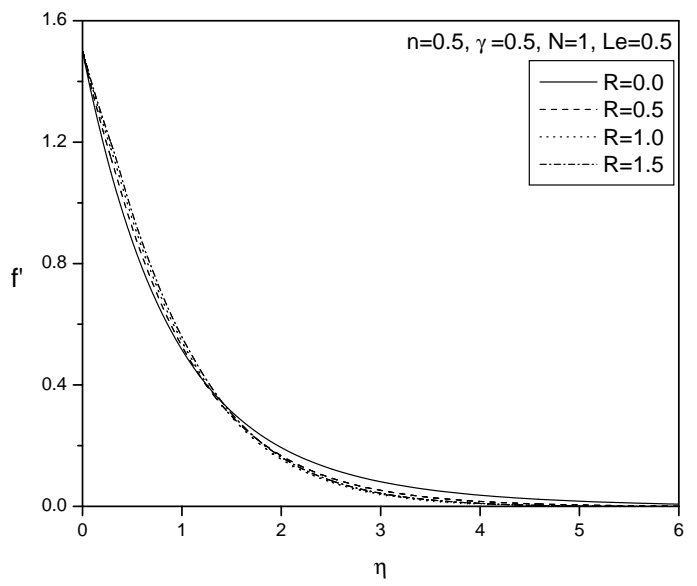

(a) Velocity profile

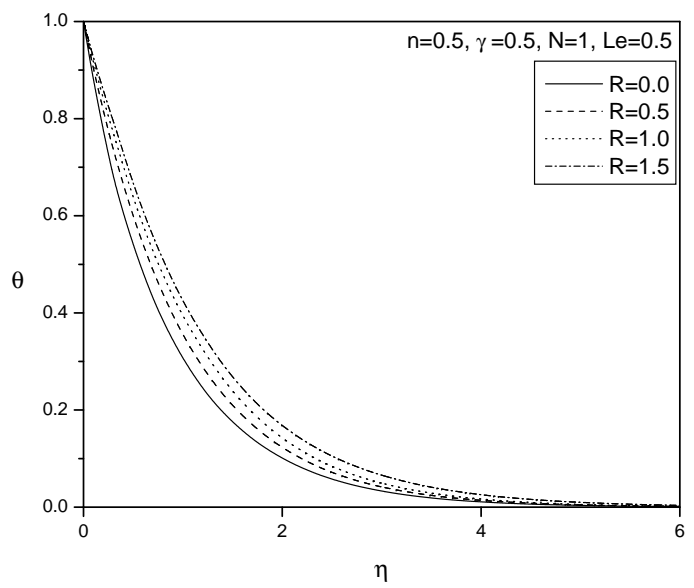

(b) Temperature profile

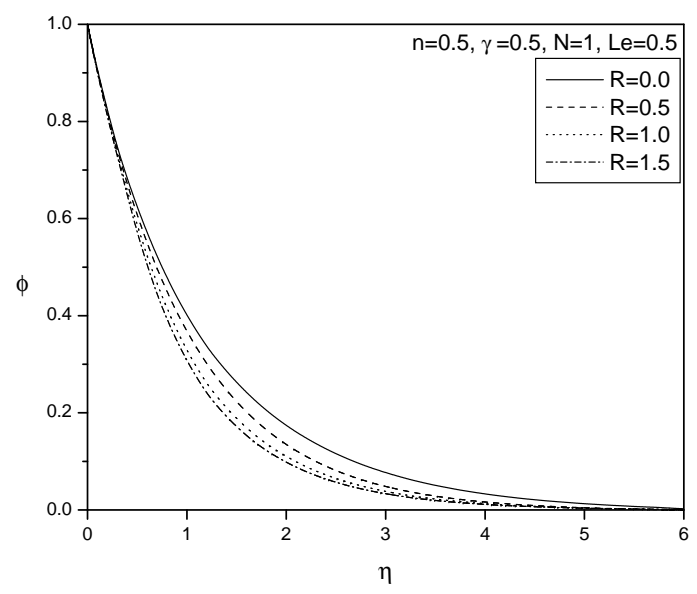

(c) Concentration profile

Fig. 2 Velocity,Temperature and Concentration profiles for various values of $R$ for pseudo-plastic fluids.

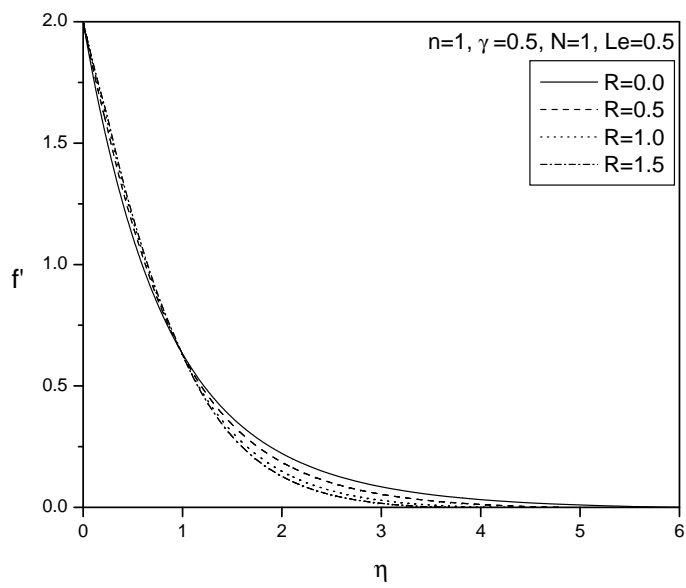

(a) Velocity profile

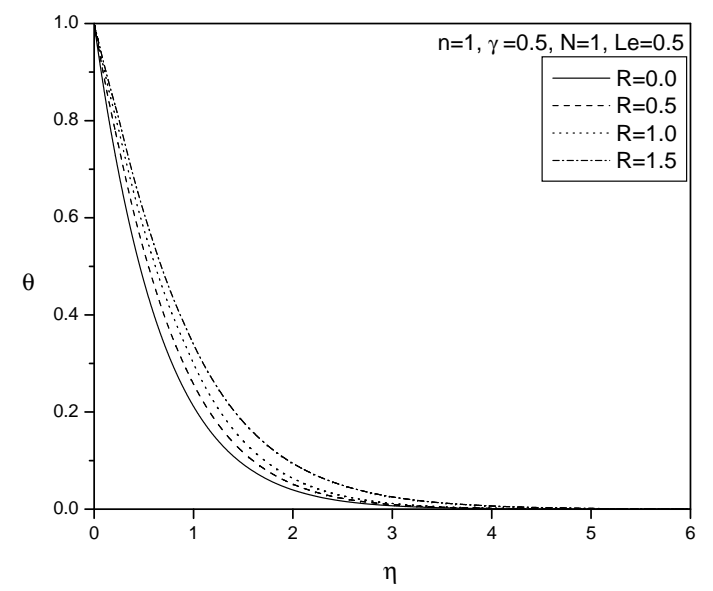

(b) Temperature profile

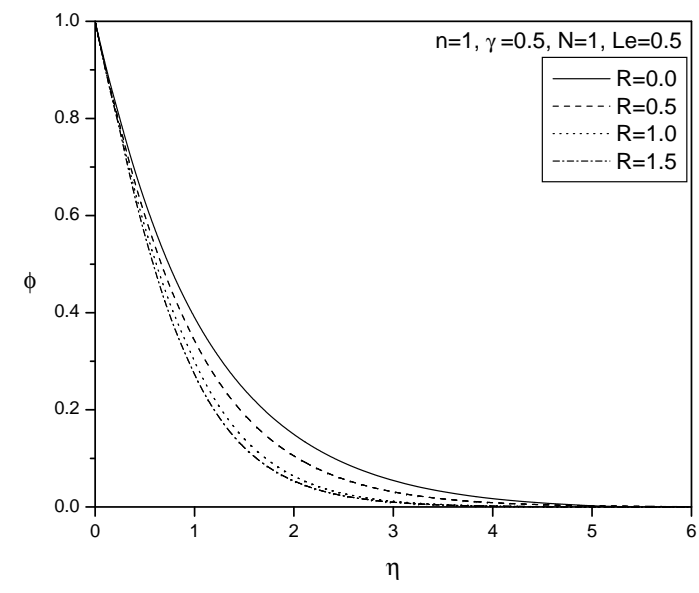

(c) Concentration profile

Fig. 3 Velocity,Temperature and Concentration profiles for various values of $R$ for Newtonian fluids. 


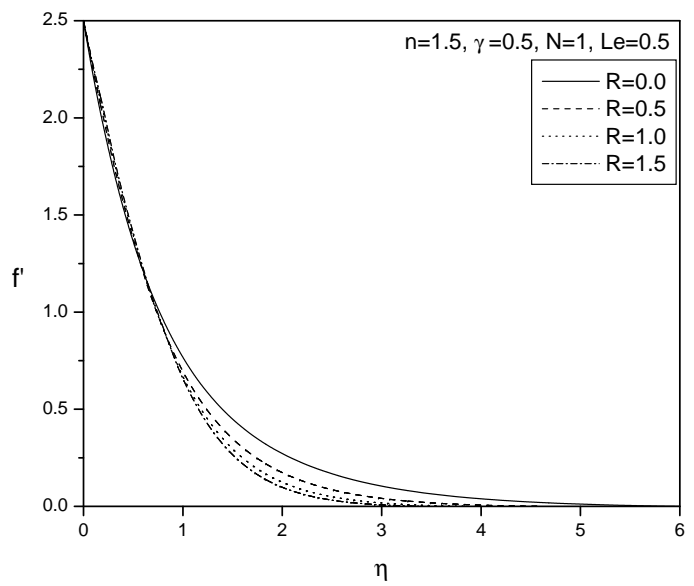

(a) Velocity profile

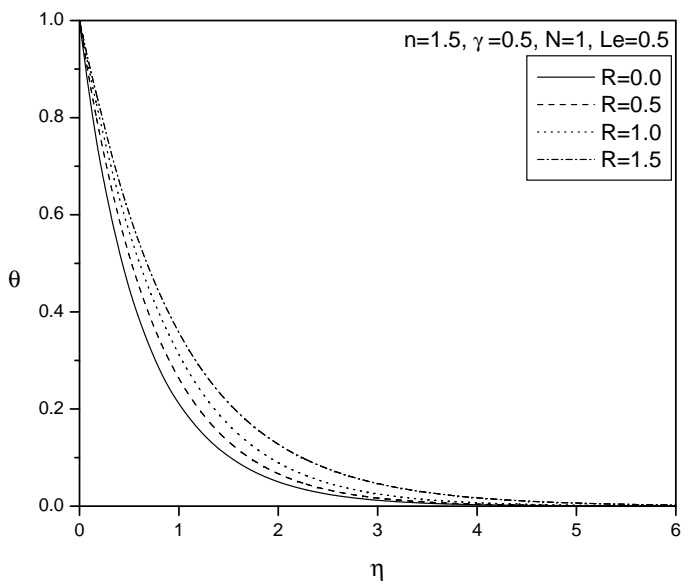

(b) Temperature profile

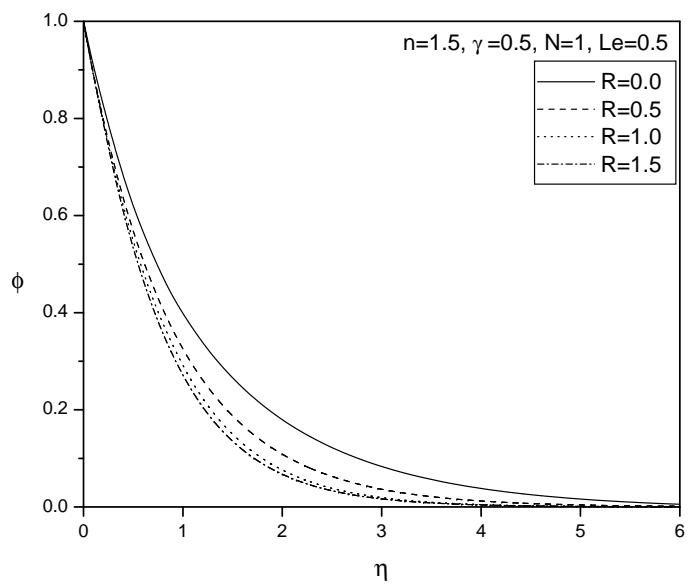

(c) Concentration profile

Fig. 4 Velocity,Temperature and Concentration profiles for various values of $R$ for dilatant fluids.

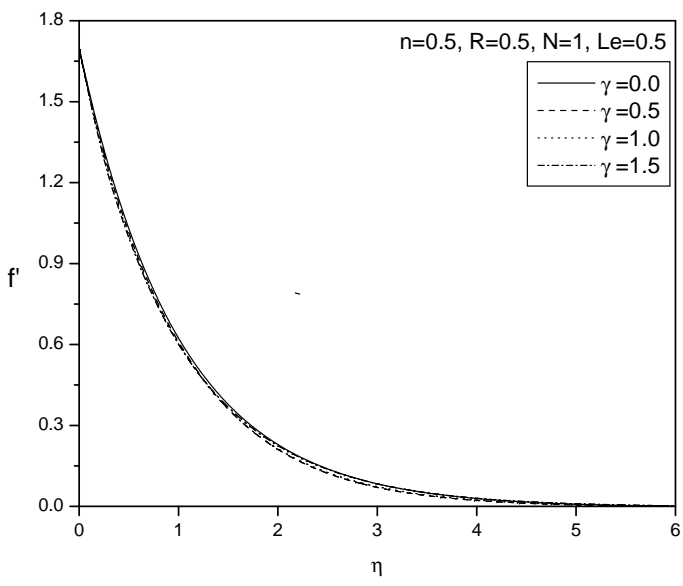

(a) Velocity profile

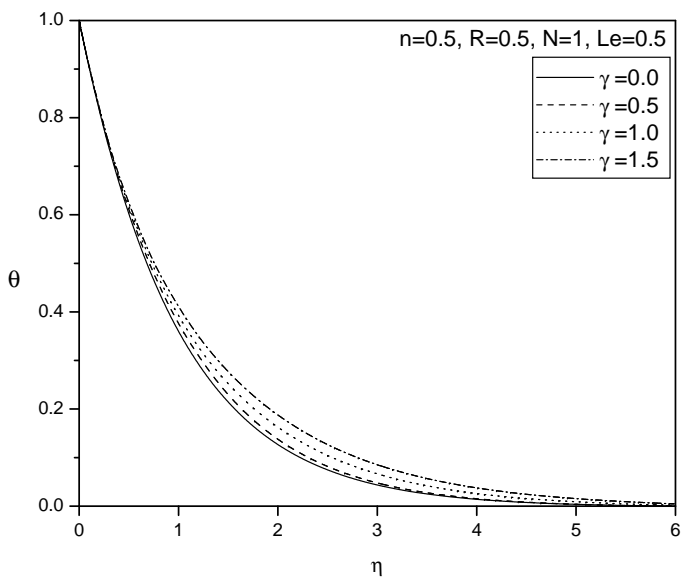

(b) Temperature profile

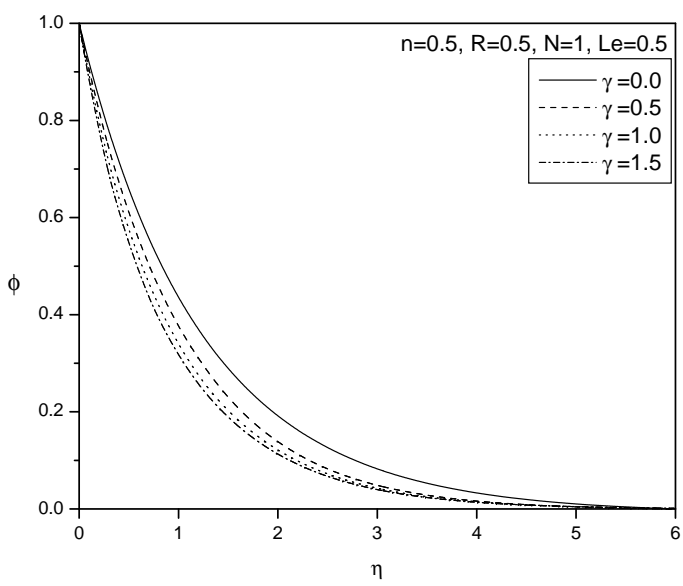

(c) Concentration profile

Fig. 5 Velocity,Temperature and Concentration profiles for various values of $\gamma$ for pseudo-plastic fluids. 
ter. It is found from Fig.5(b) that the temperature of the fluid is increased with the increase in the value of the chemical reaction parameter. It is seen from Fig.5(c) that the concentration of the fluid is decreased with increase in the value of the chemical reaction parameter. Higher values of $\gamma$ amount to a fall in the chemical molecular diffusivity, i.e., less diffusion. Therefore, they are obtained by species transfer. An increase in $\gamma$ will suppress species concentration. The concentration distribution decreases at all points of the flow field with the increase in the reaction parameter.

The variation of the non-dimensional velocity $f^{\prime}(\eta)$, temperature $\theta(\eta)$ and concentration $\phi(\eta)$ are plotted for $N=1, L e=0.5, R=0.5$ with varying chemical reaction parameter by considering Newtonian fluids with $n=1.0$ is shown in Figs.6(a)-6(c). It is observed from Fig.6(a) that the velocity of the fluid decreased with increasing the value of the chemical reaction parameter. Fig.6(b) demonstrates that the increase in the value of the chemical reaction parameter increases the temperature of the fluid in the medium. It is seen from Fig.6(c) that the concentration of the fluid is decreased by increasing the value of the chemical reaction parameter.

The effect chemical reaction parameter on the non-dimensional velocity $f^{\prime}(\eta)$, temperature $\theta(\eta)$ and concentration $\phi(\eta)$ for $N=1, L e=$ $0.5, R=0.5$ is depicted in Figs.7(a)-7(c) in the presence of dilatant fluids with $n=1.5$. It is observed from Fig.7(a) that the fluid velocity is decreased with increase in the value of chemical reaction parameter. It is seen from Fig.7(b) that the temperature of the fluid in the medium is increased with increase in the value of the chemical reaction parameter. It is found from Fig.7(c) the concentration of the fluid is decreased by increasing the value of the chemical reaction parameter.

The non-dimensional velocity $f^{\prime}(\eta)$, temperature $\theta(\eta)$ and concentration $\phi(\eta)$ for $N=1.0, L e=1.5, \gamma=0.5, R=0.5$ with a variation in power law index parameter is plotted in Figs.8(a)-8(c). It is observed from Fig.8(a) that the fluid velocity is increased with increase in the value of the power law index parameter. The effect of the increasing values of the power law index $n$ is to increase the horizontal boundary layer thickness. That is, the thickness is much smaller for shear thinning (pseudo plastic; $n<1$ ) fluids than that of shear thickening (dilatants; $n>1$ ) fluids. In the case of a shear thinning fluid $(n<1)$, the shear rates near the walls are higher than those for a Newtonian fluid. It can be seen from Figs.8(b) and 8(c) that the temperature and concentration of the fluid is increased with increase in the value of the power law index parameter. Increasing the values of the power law index leads to increase the thermal and solutal boundary layer thickness.

Table 1 shows the effects of $n, L e, R, \gamma$ and $N$ on the non-dimensional heat and mass transfer coefficients. It is seen from this table that both the heat and mass transfer rates increase with increasing power law index $n$. The Lewis number (diffusion ratio) is the ratio of Schmidt number $\left(\nu / D_{m}\right)$ and Prandtl number $\left(\nu / \alpha_{m}\right)$. As the Lewis number increases i.e. Schmidt number increases ( or Prandtl number decreases) Nusselt number is decreasing while the Sherwood number is increasing. The Schmidt number quantifies the relative effectiveness of momentum and mass transport by diffusion in the hydrodynamic (velocity) and concentration (species) boundary layers. Hence the rate of mass transfer is increased with the increase in Schmidt number or Lewis number. Similarly, decrease in Prandtl number i.e. increase in Lewis number is equivalent to increasing the thermal conductivities, and therefore heat is able to diffuse away from the heated plate more rapidly. Hence the rate of heat transfer is reduced. The heat transfer rate is decreasing for increasing values of radiation parameter but the mass transfer rate is increasing. The chemical reaction parameter decreases the heat transfer coefficient but increases the mass transfer rate. Increase in the values of $\gamma$ implies more interaction of species concentration with the momentum boundary layer and less interaction with the thermal boundary layer. Hence, chemical reaction parameter has more significant effect on Sherwood number than it does on Nusselt number. There is increase in both the heat and mass transfer rates with increase in the buoyancy ratio.

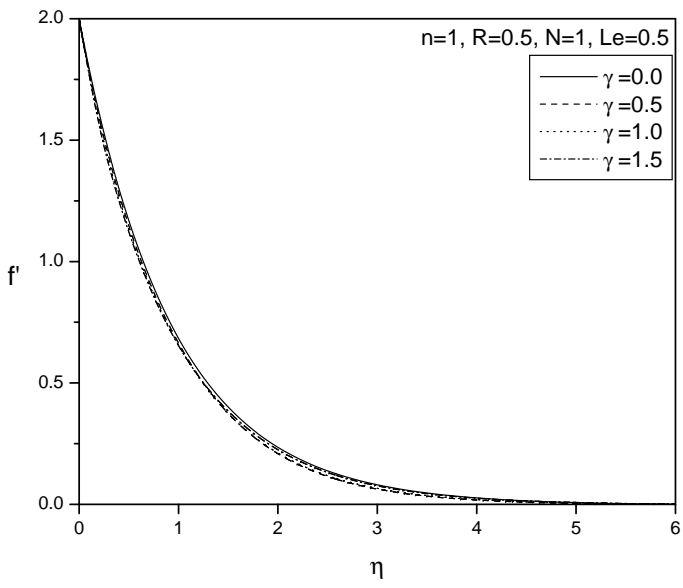

(a) Velocity profile

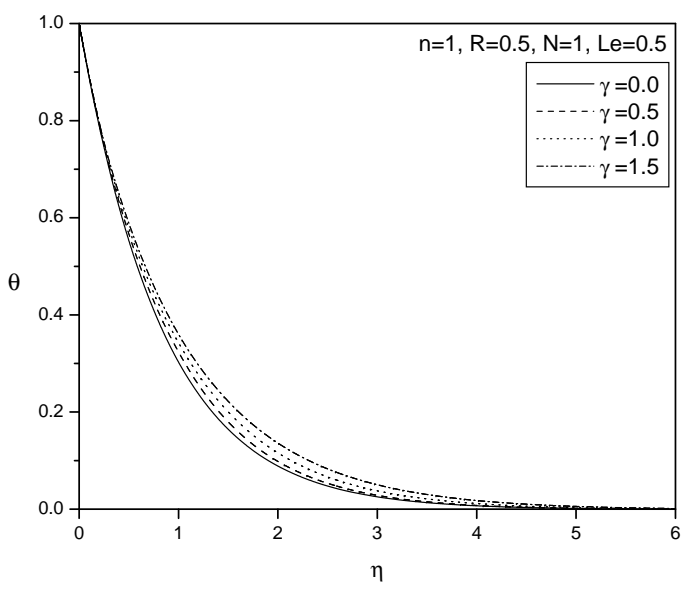

(b) Temperature profile

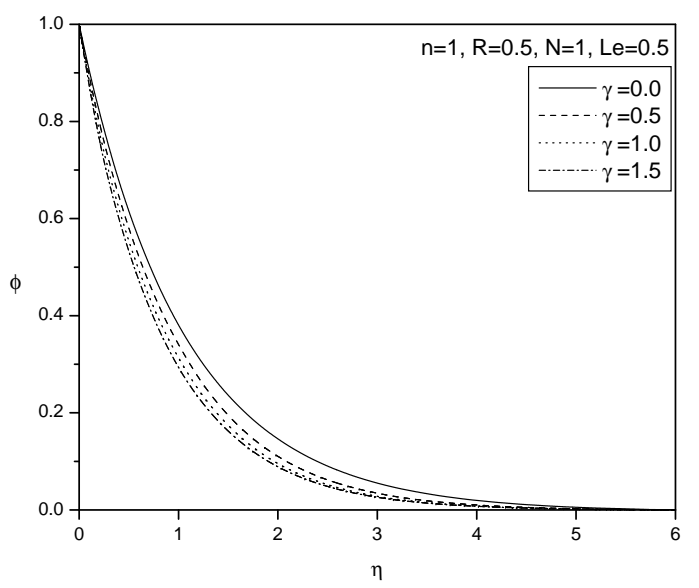

(c) Concentration profile

Fig. 6 Velocity,Temperature and Concentration profiles for various values of $\gamma$ for Newtonian fluids. 


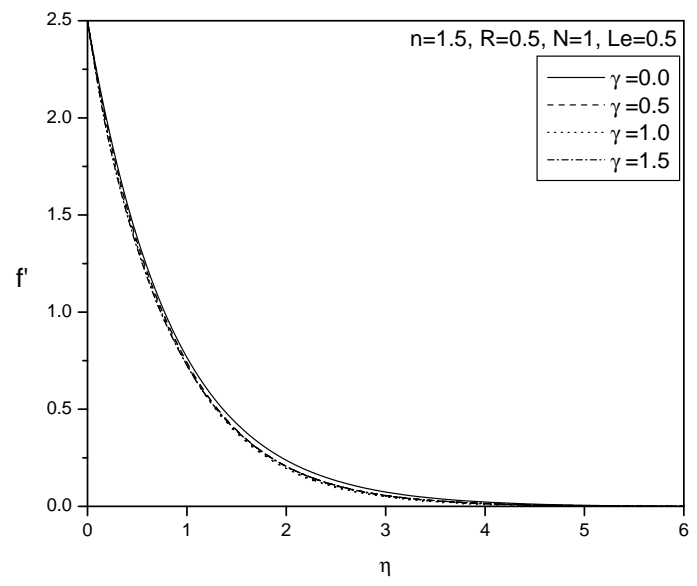

(a) Velocity profile

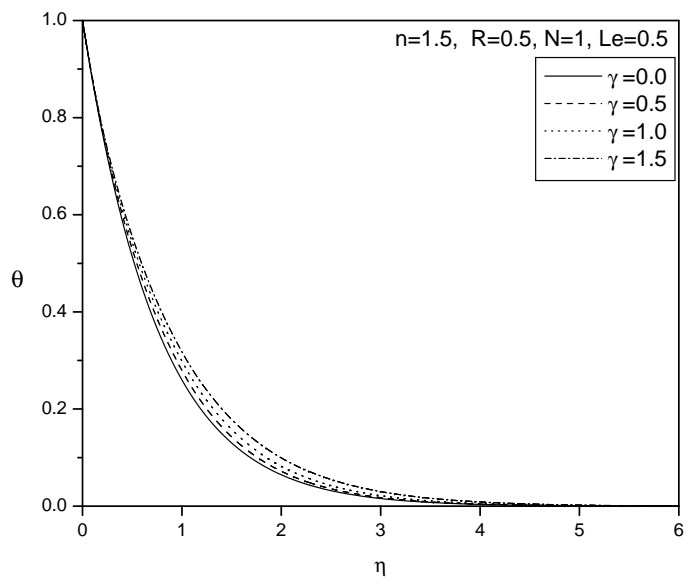

(b) Temperature profile

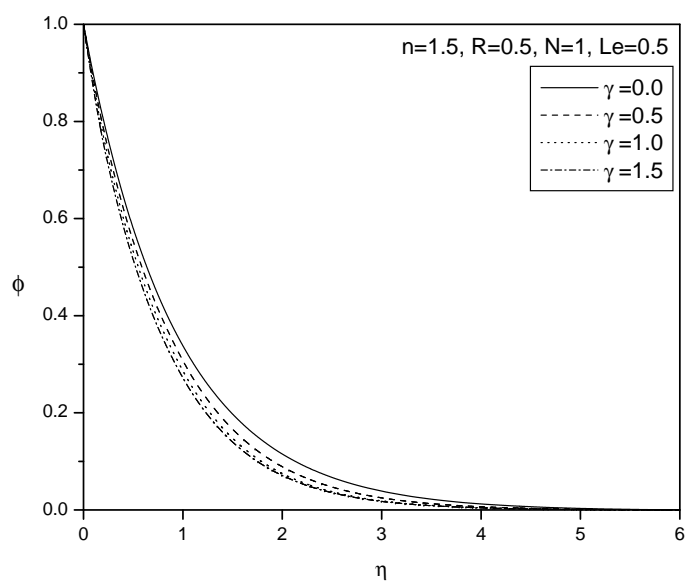

(c) Concentration profile

Fig. 7 Velocity,Temperature and Concentration profiles for various values of $\gamma$ for dilatant fluids.

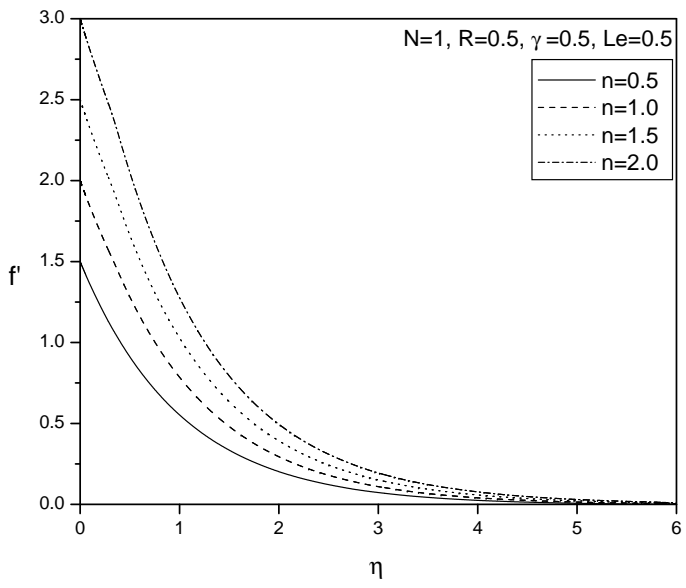

(a) Velocity profile

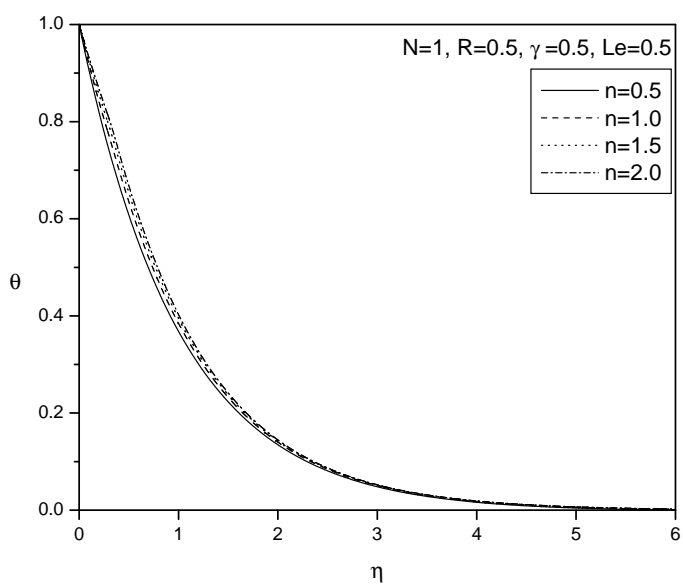

(b) Temperature profile

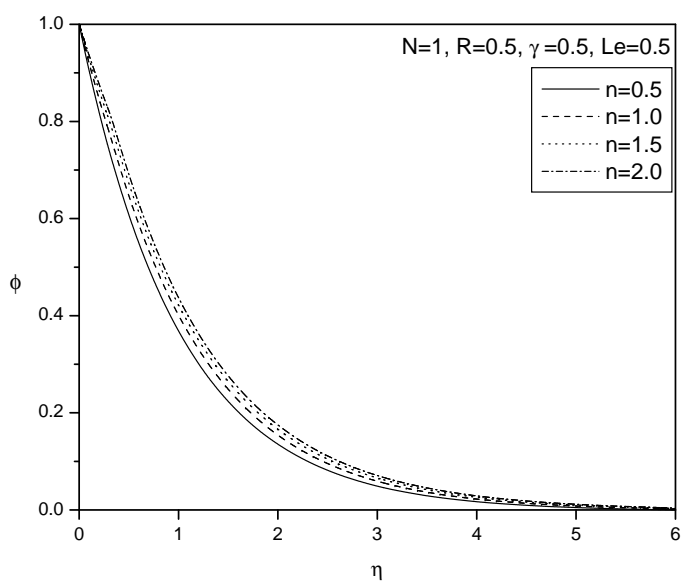

(c) Concentration profile

Fig. 8 Velocity,Temperature and Concentration profiles for various values of Power-law index $(n)$. 
Table 1 Variation of non-dimensional heat and mass transfer coefficients for various values of $n, L e, R, \gamma$ and $N$.

\begin{tabular}{ccccccc}
\hline$n$ & $L e$ & $R$ & $\gamma$ & $N$ & $\frac{N u_{x}}{B x}=-\theta^{\prime}(0)$ & $\frac{S h_{x}}{B x}=-\phi^{\prime}(0)$ \\
\hline 0.0 & 1 & 0.5 & 0.5 & 1 & 0.819658 & 0.771853 \\
0.5 & 1 & 0.5 & 0.5 & 1 & 1.001512 & 1.000471 \\
1.0 & 1 & 0.5 & 0.5 & 1 & 1.151338 & 1.190781 \\
1.5 & 1 & 0.5 & 0.5 & 1 & 1.281963 & 1.357628 \\
2.0 & 1 & 0.5 & 0.5 & 1 & 1.399380 & 1.508127 \\
\hline 0.5 & 0.0 & 0.5 & 0.5 & 1 & 1.073281 & 0.166667 \\
0.5 & 0.5 & 0.5 & 0.5 & 1 & 1.024082 & 0.709265 \\
0.5 & 1.0 & 0.5 & 0.5 & 1 & 1.000527 & 1.000153 \\
0.5 & 1.5 & 0.5 & 0.5 & 1 & 0.985709 & 1.204263 \\
0.5 & 2.0 & 0.5 & 0.5 & 1 & 0.975334 & 1.361573 \\
\hline 0.5 & 1 & 0.0 & 0.5 & 1 & 1.210337 & 1.437245 \\
0.5 & 1 & 0.5 & 0.5 & 1 & 0.971526 & 1.466103 \\
0.5 & 1 & 1.0 & 0.5 & 1 & 0.831683 & 1.483859 \\
0.5 & 1 & 1.5 & 0.5 & 1 & 0.737831 & 1.496092 \\
0.5 & 1 & 2.0 & 0.5 & 1 & 0.669629 & 1.505127 \\
\hline 0.5 & 1 & 0.5 & 0.0 & 1 & 0.982493 & 1.265102 \\
0.5 & 1 & 0.5 & 0.5 & 1 & 0.971526 & 1.466103 \\
0.5 & 1 & 0.5 & 1.0 & 1 & 0.963397 & 1.637643 \\
0.5 & 1 & 0.5 & 1.5 & 1 & 0.956913 & 1.790464 \\
0.5 & 1 & 0.5 & 2.0 & 1 & 0.951518 & 1.929889 \\
\hline 0.5 & 1 & 0.5 & 0.5 & 0.6 & 1.113030 & 1.148824 \\
0.5 & 1 & 0.5 & 0.5 & 0.7 & 1.117599 & 1.150429 \\
0.5 & 1 & 0.5 & 0.5 & 0.8 & 1.123225 & 1.156633 \\
0.5 & 1 & 0.5 & 0.5 & 0.9 & 1.135297 & 1.171184 \\
0.5 & 1 & 0.5 & 0.5 & 1.0 & 1.151338 & 1.190781 \\
\hline
\end{tabular}

\section{CONCLUSIONS}

In this paper, natural convection heat and mass transfer along a vertical plate embedded in a power-law fluid saturated Darcy porous medium in presence of radiation and chemical reaction has been considered. The wall is maintained at variable temperature and concentration $T_{w}(x)$ and $C_{w}(x)$, respectively. It can be concluded from the present analysis that the higher values of radiation parameter result in higher velocity and temperature distributions but lower concentration distribution. An increase in the values of the chemical reaction parameter results in lower velocity and concentration distributions but higher temperature distribution. Also, the higher values of the power-law index parameter result in higher velocity, temperature and concentration distributions within the boundary layer.

\section{NOMENCLATURE}

A a dimensional constant.

$B \quad$ a dimensional constant.

a a constant.

$b \quad$ a constant.

$l \quad$ Temperature variation index at wall.

T Temperature.

$C \quad$ Concentration.

$g \quad$ Gravitational acceleration.

$T_{\infty} \quad$ Ambient temperature.

$K \quad$ Darcy Permeability.

$k_{1} \quad$ Rate of chemical reaction.

$k \quad$ Thermal conductivity.

$q_{y}^{r} \quad$ Radiative heat flux.

$C_{p} \quad$ Specific heat at constant pressure.

$C_{s} \quad$ Concentration susceptibility.

$\alpha_{m} \quad$ Thermal diffusivity.

$D_{m} \quad$ Mass diffusivity.

$\gamma \quad$ Chemical reaction parameter.
$R \quad$ Radiation parameter

$\sigma \quad$ Stefan-Boltzman constant.

Le Lewis number.

$N \quad$ Buoyancy ratio.

$n \quad$ Power-law index.

$\beta_{T} \quad$ Coefficient of thermal expansion.

$\beta_{C} \quad$ Coefficient of concentration expansion.

$N u_{x} \quad$ Nusselt number.

$S h_{x} \quad$ Sherwood number.

$\nu \quad$ Kinematic viscosity.

$\phi \quad$ Dimensionless concentration.

$\theta \quad$ Dimensionless temperature.

$u, v \quad$ Darcian Velocity components in $\mathrm{x}$ and $\mathrm{y}$ directions.

$x, y \quad$ Co-ordinates along and normal to the plate.

$\eta \quad$ Similarity variable.

$\psi \quad$ Stream function.

Subscripts

$w \quad$ Wall condition.

$\infty \quad$ Ambient condition.

T Temperature.

Superscripts

1 Differentiation with respect to $\eta$.

\section{REFERENCES}

Aouachria, Z., Rouichi, F., and Haddad, H. 2012, "Double diffusion effects on convection in flow on vertical plate imbedded in porous media", Frontiers in Heat and Mass Transfer, 3, 023004 , http://dx.doi.org/10.5098/hmt.v3.2.3004

Chamkha, A. J., Aly,A. M., and Mansour, M. A. ,2010, "Unsteady natural convective power-law fluid flow past a vertical plate embedded in a non-Darcian porous medium in the presence of a homogeneous chemical reaction", Nonlinear Analysis: Modelling and Control 15(2), 139-154.

Elgazery, N. S., 2008, "Transient analysis of heat and mass transfer by natural convection in power law fluid past a vertical plate immersed in a porous medium (numerical study)", Applications and Applied Mathematics, 3(2), 2008, 267-285.

Ghosh, S.K. and I. Pop, 2007, "Thermal radiation of an optically thick gray gas in the presence of indirect natural convection", Int. J. Fluid Mech. Res. 34(6), 515-520,

http://dx.doi.org/10.1615/InterJFluidMechRes.v34.i6

Gaied, A. S. M. and Eid, M. R. ,2011, “ Natural convection of non-Newtonian power-law fluid over axisymmetric and two-dimensional bodies of arbitrary shape in fluid-saturated porous media", Applied Mathematics and Mechanics 32, 179-188,

http://dx.doi.org/10.1007/s10483-011-1404-6

Hayat, T., Abbas, Z., Pop I., and Asghar, S. ,2010, "Effects of radiation and magnetic field on the mixed convection stagnation-point flow over a vertical stretching sheet in a porous medium", Int. J. Heat and Mass Transfer 53, 466-474,

http://dx.doi.org/10.1016/j.ijheatmasstransfer.2009.09.010

Hossain, M.A., Khanafer, K. and Vafai, K., 2001, "The effect of radiation on free convection flow of fluid with variable viscosity from a porous vertical plate", Int. J. Therm. Sci., 40, 115-124.

http://dx.doi.org/10.1016/S1290-0729(00)01200-X

Kairi, R. R and Murthy, P. V. S. N. ,2009, "Free Convection in a 
Thermally Stratified Non-Darcy Porous Medium Saturated with a Non-Newtonian Fluid", Int. J Fluid Mech. Res. 36(5),

http://dx.doi.org/ 10.1615/InterJFluidMechRes.v36.i5.20

Kim, G. B and Hyun, J. M. ,2004, "Buoyant convection of powerlaw fluid in an enclosure filled with heat generating porous media", Numerical Heat Transfer Part A: Applications 45, 569-582, http://dx.doi.org/10.1080/10407780490277572

Kuznetsov, A. V. and Nield, D. A., 2010, "The cheng-minkowycz problem for cellular porous materials: Effect of temperature-dependent conductivity arising from radiative transfer", Int. J. of Heat and Mass Transfer, 53, pp. 2676-2679,

http://dx.doi.org/10.1016/j.ijheatmasstransfer.2010.02.042

Nakayama, A. ,1993, "Free convection from a horizontal line heat source in a power-law fluid-saturated porous medium", Int. J. of Heat and Fluid Flow 14, 279-283,

http://dx.doi.org/10.1016/0142-727X(93)90059-V

Nield, D. A and Bejan, A. 2006, Convection in porous media (Springer-Verlag, 3rd Edition, New York).

ISBN: 0387984437

Orhan Aydn, A. K. ,2008, "Radiation Effect on MHD Mixed Convection Flow about a Permeable Vertical Plate", Heat Mass Transfer 45, 239-246,

http://dx.doi.org/10.1007/s00231-008-0428-y

Postelnicu, A. ,2007, "Influence of Chemical Reaction on Heat and Mass Transfer by Natural Convection from Vertical Surfaces in Porous
Media Considering Soret and Dufour Effects",Heat Mass Transfer 43, 595-602,

http://dx.doi.org/10.1007/s00231-006-0132-8

Prabhu, K. K. S, Kandasamy, R., and Periasamy, K. ,2005, "Chemical Reaction, Heat and Mass Transfer on MHD Flow Over a Vertical Stretching Surface with Heat Source and Thermal Stratification Effects", Int. J. Heat Mass Transfer 48, 4557-4561,

http://dx.doi.org/10.1016/j.ijheatmasstransfer.2005.05.006

Rami, Y. J and Arun, S. M. ,2000, "Free convection heat and mass transfer of non-Newtonian power law fluids with yield stress from a vertical flat plate in a saturated porous media", Int. Commun. Heat Mass Transfer 27, 485-494, http://dx.doi.org/10.1016/S0735-1933(00)00131-7

Sahu, A. K and Mathur, M. N. ,1996, "Free convection in boundary layer flows of power law fluids past a vertical flat plate with suction/injection", Indian Journal of Pure and Applied Mathematics 27, 931-941.

Salem, A. M. ,2006, "Coupled Heat and Mass Transfer in DarcyForchheimer Mixed Convection from a Vertical Flat Plate Embedded in a Fluid-Saturated Porous Medium under the Effects of Radiation and Viscous Dissipation", Journal of the Korean Physical Society 48, 409-413.

Sparrow, E. M and Cess, R. D. ,1978, Radiation Heat Transfer (Hemispherer publi Corp Washington). 